Preparation and stimulation of Tcells. Isolation of splenocytes and enriched human $\mathrm{T}$ cells and their stimulation with concanavalin $\mathrm{A}$ and PHA were done as described $^{2,30}$.

Apoptosis and cell proliferation assays. Reduced cell proliferation or apoptosis as a result of death receptor stimulation or overexpression was measured using the Celltiter $96 \mathrm{AQ}$ proliferation assay (Promega) and the cell death detection ELISA (Boehringer Mannheim), respectively, as previously described $^{3}$.

Protease activity assays. Flag fusion proteins of the caspase homology region of FLIP $_{\mathrm{L}}$ and FLICE were generated in bacteria using modified pQE16 (Qiagen) bacterial expression vectors. Protease activity was determined on fluorogenic substrates as described ${ }^{7}$.

Chromosomal localization. A sequence-tagged site ${ }^{11}$ (A008B37) corresponding to FLIP maps to human chromosome 2 in the interval between the markers D2S116-D2S307. On the cytogenetic map, this interval corresponds to 2q33.

Received 19 May; accepted 11 June 1997.

1. Nagata, S. Apoptosis by death factor. Cell 88, 355-365 (1997)

2. Klas, C., Debatin, K. M., Jonker, R. R. \& Krammer, P. H. Activation interferes with the APO-1 pathway in mature human T cells. Int. Immunol. 5, 625-630 (1993).

. Thome, M. et al. Viral FLICE-inhibitory proteins (FLIPs) prevent apoptosis induced by death receptors. Nature 386, 517-521 (1997)

4. Boldin, M. P. et al. A novel protein that interacts with the death domain of Fas/APO1 contains a sequence motif related to the death domain. J. Biol. Chem. 270, 7795-7798 (1995).

5. Chinnaiyan, A. M., O'Rourke, K., Tewari, M. \& Dixit, V. M. FADD, a novel death domain-containing protein, interacts with the death domain of Fas and initiates apoptosis. Cell 81, 505-512 (1995).

6. Muzio, M. et al. FLICE, a novel FADD-homologous ICE/CED-3-like protease, is recruited to the CD95 (Fas/APO-1) death-inducing signaling complex. Cell 85, 817-827 (1996)

. Boldin, M. P., Goncharov, T. M., Goltsev, Y. V. \& Wallach, D. Involvement of MACH, a novel MORT1/ FADD-interacting protease, in Fas/APO-1- and TNF receptor-induced cell death. Cell 85, 803-815 (1996).

8. Bertin, J. et al. Death effector domain-containing herpesvirus and poxvirus proteins inhibit both Fasand TNFR1-induced apoptosis. Proc. Natl Acad. Sci. USA 94, 1172-1176 (1997).

9. Bucher, P., Karplus, K., Moeri, N. \& Hofmann, K. A flexible search technique based on generalized profiles. Comput. Chem. 20, 3-24 (1996).

10. Fernandes-Alnemri, T. et al. In vitro activation of CPP32 and Mch3 by Mch4, a novel human apoptotic cysteine protease containing two FADD-like domains. Proc. Natl Acad. Sci. USA 93, 7464 7469 (1996).

11. Schuler, G. D. et al. A gene map of the human genome. Science 274, 540-546 (1996).

12. Walker, N. P. C. et al. Crystal structure of the cysteine protease interleukin-1- $\beta$-converting enzyme-a (p20/p10)(2) homodimer. Cell 78, 343-352 (1994).

13. Wilson, K. P. et al. Structure and mechanism of interleukin-1 $\beta$ converting enzyme. Nature 370, 270 275 (1994)

14. Vincenz, C. \& Dixit, V. M. Fas-associated death domain protein interleukin-1 $\beta$-converting enzyme 2 (FLICE2), an ICE/Ced-3 homologue, is proximally involved in CD95- and p55-mediated death signaling. J. Biol. Chem. 272, 6578-6583 (1997).

15. Kischkel, F. C. et al. Cytotoxicity-dependent Apo-1 (Fas/CD95)-associated proteins form a deathinducing signaling complex (DISC) with the receptor. EMBO J. 14, 5579-5588 (1995).

16. Gu, Y. et al. Interleukin-1 $\beta$ converting enzyme requires oligomerization for activity of processed forms in vivo. EMBO J. 14, 1923-1931 (1995).

17. Medema, J. P. et al. FLICE is activated by association with the CD95 death-inducing signaling complex (DISC). EMBO J. 16, 2794-2804 (1997).

18. Bump, N. J. et al. Inhibition of ICE family proteases by baculovirus antiapoptotic protein $\mathrm{p} 35$. Science 269, 1885-1888 (1995)

19. Xue, D. \& Horvitz, H. R. Inhibition of the Caenorhabditis elegans cell-death protease CED-3 by a CED3 cleavage site in baculovirus p35 protein. Nature 377, 248-251 (1995).

20. Bodmer, J. L. et al. TRAMP, a novel apoptosis-mediating receptor with sequence homology to tumor necrosis factor receptor 1 and Fas(Apo-1/CD95). Immunity 6, 79-88 (1997).

21. Marsters, S. A. et al. Apo-3, a new member of the tumor necrosis factor receptor family, contains a death domain and activates apoptosis and NF-кB. Curr. Biol. 6, 1669-1676 (1996).

22. Kitson, J. et al. A death-domain-containing receptor that mediates apoptosis. Nature 384, 372-375 (1996).

23. Yu, G. L. et al. Signal transduction by DR3, a death domain-containing receptor related to TNFR-1 and CD95. Science 274, 990-992 (1996).

24. Tartaglia, L. A., Rothe, M., Hu, Y. F. \& Goeddel, D. V. Tumor necrosis factor's cytotoxic activity is signaled by the p55 TNF receptor. Cell 73, 213-216 (1993).

25. Pan, G. et al. The receptor for the cytotoxic ligand TRAIL. Science 276, 111-113 (1997).

26. Peter, M. E. et al. Resistance of cultured peripheral T cells towards activation-induced cell death involves a lack of recruitment of FLICE (MACH/caspase 8) to the CD95 death-inducing signaling complex. Eur. J. Immunol. 27, 1207-1212 (1997).

27. Hahne, M. et al. Melanoma cells express Fas(Apo-1/CD95) ligand: Implications for tumor immune escape. Science 267, 1363-1366 (1996)

28. Nagata, S. \& Golstein, P. The Fas death factor. Science 267, 1449-1456 (1995).

29. Francis, M. J. et al. Immunological evaluation of the multiple antigen peptide (MAP) system using the major immunogenic site of foot-and-mouth disease virus. Immunology 73, 249-254 (1991).

30. Lowin, B., Hahne, M., Mattmann, C. \& Tschopp, J. Cytolytic T-cell cytoxocity is mediated through perforin and Fas lytic pathways. Nature 370, 650-652 (1994).

Acknowledgements. We thank S. Belli for reading this manuscript; C. Servi for peptide synthesis; and R. Bullani, S. Hertig, M. Rousseaux and T. Bornand for technical assistance. This work was supported by grants of the Swiss National Science Foundation (J.T.) and the European Molecular Biology Organisation (to M.T.).

Correspondence and requests for material should be addressed to J.T. (e-mail: jurg.tschopp@ib.unil.ch).

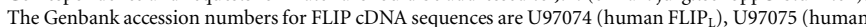
FLIP $_{S}$ ), and U97076 (mouse FLIP $_{\mathrm{L}}$ ).

\section{Tom5 functionally links mitochondrial preprotein receptors to the general import pore}

\author{
Klaus Dietmeier*, Angelika Hönlinger*, Ulf Bömer, \\ Peter J. T. Dekker, Christoph Eckerskorn $\dagger$, \\ Fritz Lottspeich $†$, Michael Kübrich \& Nikolaus Pfanner
}

Institut für Biochemie und Molekularbiologie, Universität Freiburg,

Hermann-Herder-Strasse 7, D-79104 Freiburg, Germany

$\dagger$ Max-Planck-Institut für Biochemie, D-82152 Martinsried, Germany

* These authors contributed equally to this study.

Most mitochondrial proteins are synthesized as preproteins on cytosolic polysomes and are subsequently imported into the organelle ${ }^{1-3}$. The mitochondrial outer membrane contains a multisubunit preprotein translocase (Tom) which has receptors on the cytosolic side and a general import pore (GIP) in the membrane. Tom20-Tom 22 and Tom70-Tom37 function as import receptors ${ }^{4-7}$ with a preference for preproteins that have amino-terminal presequences or internal targeting information, respectively. Tom 40 is an essential constituent of the $\mathrm{GIP}^{8,9}$, whereas Tom 6 and Tom 7 modulate the assembly and dissociation of the Tom machinery ${ }^{10,11}$. Here we report the identification of Tom5, a small subunit that has a crucial role importing preproteins destined for all four mitochondrial subcompartments. Tom 5 has a single membrane anchor and a cytosolic segment with a negative net charge, and accepts preproteins from the receptors and mediates their insertion into the GIP. We conclude that Tom5 represents a functional link between surface receptors and GIP, and is part of an 'acid chain's that guides the stepwise transport of positively charged mitochondrial targeting sequences.

For the preparation of Tom machinery, digitonin-lysed mitochondria from the yeast Saccharomyces cerevisiae were subjected to a coprecipitation with antibodies directed against Tom40. A noncharacterized band (labelled 'Tom5' in Fig. 1a, lane 2) was subjected to $\mathrm{N}$-terminal sequencing (Fig. $1 \mathrm{~b}$, sequence II). The sequence matched to a previously unknown open reading frame (ORF) on chromosome XVI (cosmid 9659, nucleotides 6,512-6,661), encoding a 50-residue protein of relative molecular mass $\left(M_{\mathrm{r}}\right) 5.98 \mathrm{~K}$ (Fig. 1b) which we term Tom5. Its sequence does not reveal significant homology to any known protein. The C-terminal half of Tom5 contains a hydrophobic segment (residues 27-45) of sufficient length to function as a membrane anchor. This segment is flanked by positively charged residues that may interact with negatively charged headgroups of membrane phospholipids. The $\mathrm{N}$-terminal portion of Tom 5 carries a negative net charge (Fig. 1b).

Pure outer-membrane vesicles ${ }^{12,13}$ contained a prominent band with an electrophoretic mobility like that of Tom5 (Fig. 1a, lane 1), and N-terminal amino-acid sequencing confirmed that it was indeed Tom5 (Fig. 1b, sequence I). An antiserum directed against an N-terminal peptide of Tom5 was generated (Fig. 1a, lane 3). To assess whether Tom 5 is an integral membrane protein, mitochondria were treated with sodium carbonate at $\mathrm{pH} 11.5$ (ref. 11). This treatment allows the extraction of soluble and peripheral membrane proteins (Fig. 1c, columns 3 and 5) but leaves integral membrane proteins in the membrane sheets (Fig. 1c, columns 2 and 4). Tom5 was fully resistant to extraction by sodium carbonate (Fig. 1c, column 1), indicating that it is an integral membrane protein. Tom 5 was also resistant to treatment of mitochondria with trypsin at all concentrations tested (Fig. 1d, lanes 2-6), even after lysis of the mitochondria with Triton X-100 (Fig. 1d, lane 7). Treatment of 
mitochondria with proteinase $\mathrm{K}$, however, led to complete loss of material reacting with the anti-Tom 5 antibodies at low concentrations of the protease (Fig. 1d, lanes 9-13), demonstrating that the $\mathrm{N}$ terminus was digested. The marker proteins cytochrome $b_{2}$ of the intermembrane space and the ADP/ATP carrier of the inner membrane were not degraded (Fig. 1d, lanes 9-13), unless the mitochondrial membranes had been disrupted by detergent (Fig. 1d, lane 14), demonstrating that the outer-membrane barrier was not degraded by the protease treatment. We conclude that Tom 5 is an integral mitochondrial outer-membrane protein that exposes its $\mathrm{N}$ terminus to the cytosolic side.

The Tom machinery can be separated into two subcomplexes, a $400 \mathrm{~K}$ complex containing Tom 40 and Tom 22 , and a $120 \mathrm{~K}$ complex containing Tom70 (ref. 14). To determine which subcomplex contained Tom5, mitochondria were lysed with digitonin and protein complexes were analysed under non-denaturing conditions by blue native electrophoresis, followed by a second separation using urea-SDS-polyacrylamide gel electrophoresis. Tom5 migrated exclusively with the $400 \mathrm{~K}$ complex (Fig. 1e, top). Together with the efficient coprecipitation of Tom 5 with antibodies directed against Tom 40 (Fig. 1a, lane 2), these results indicate that Tom5 is a component of the larger Tom subcomplex.

We constructed a yeast mutant with a deletion of the coding region of the TOM5 gene. Growth of the tom $5 \Delta$ strain at $30^{\circ} \mathrm{C}$ was reduced in comparison to the wild type on both fermentable and non-fermentable carbon sources (threefold reduction of growth rate on glycerol-containing medium). At $37^{\circ} \mathrm{C}$, TOM5 is essential for viability (Fig. 2a, sectors 2 and 5). Expression of TOM5 from the centromeric vector pRS415 fully restored growth of tom $5 \Delta$ cells (Fig. 2a, sectors 3 and 6), demonstrating that the growth defects were caused solely by the deletion of TOM5. Mitochondria were isolated from wild-type and tom $5 \Delta$ cells and compared for their protein composition and ability to generate a membrane potential $(\Delta \psi)$. Except for the lack of Tom5, the protein composition of tom5 $\Delta$ mitochondria (analysed with marker proteins for all four subcompartments) was indistinguishable from that of wild-type mitochondria (Fig. 2b). When digitonin-lysed tom $5 \Delta$ mitochondria were separated by blue native electrophoresis, the stability of the $400 \mathrm{~K}$ and $120 \mathrm{~K}$ subcomplexes was not altered, as indicated by the unchanged mobility of Tom70, Tom 40 and Tom 22 (Fig. 1e, bottom). In contrast to Tom6 and Tom7 (refs 10, 11), Tom5 does not seem to modulate the stability of association of the Tom machinery. $\Delta \psi$ was assessed by use of the potentialsensitive fluorescent dye 3,3'-dipropylthiadicarbocyanine iodide $\left(\mathrm{DiSC}_{3}(5)\right)$. A decrease in fluorescence indicates the formation of a membrane potential ${ }^{15,16}$. The degree of quenching was similar for tom $5 \Delta$ and wild-type mitochondria (Fig. 2c), showing that tom $5 \Delta$ mitochondria, like wild-type mitochondria, were able to generate a membrane potential.

${ }^{35} \mathrm{~S}$-labelled mitochondrial preproteins were imported into isolated tom $5 \Delta$ and wild-type mitochondria. The following preproteins were tested: porin (from the outer mitochondrial membrane), cytochrome $c_{1}$ (intermembrane-space side of inner membrane), ADP/ATP carrier, $F_{1}$-ATPase subunit- $\beta$ (matrix side of inner membrane), a fusion protein between the presequence of $\mathrm{F}_{0^{-}}$ ATPase subunit 9 and dihydrofolate reductase (Su9-DHFR, targeted to the matrix $)^{17}$, and the $\alpha$-subunit of the mitochondrial-processing peptidase (matrix). Porin and the ADP/ATP carrier contain targeting information within the mature protein, whereas the other preproteins carry cleavable $\mathrm{N}$-terminal presequences. Import of all preproteins was strongly reduced with tom $5 \Delta$ mitochondria (Fig. 2d). We conclude that Tom5 is important for protein import in a manner independent of the type of targeting information and submitochondrial destination of preproteins.

We investigated whether Tom5 interacted with a preprotein in transit across the outer mitochondrial membrane. After accumulation of Su9-DHFR at the outer membrane of isolated mitochondria in the absence of a membrane potential ${ }^{5,2}$, the amino-reactive homo-bifunctional crosslinking reagent ethylene glycolbis(succinimidyl-succinate) (EGS) was added. Several crosslinking products were generated (Fig. 3a, lanes 3-5 and 7-9). The cleavable preprotein was crosslinked to Tom 20/Tom 22 and to Tom 40 (probably a
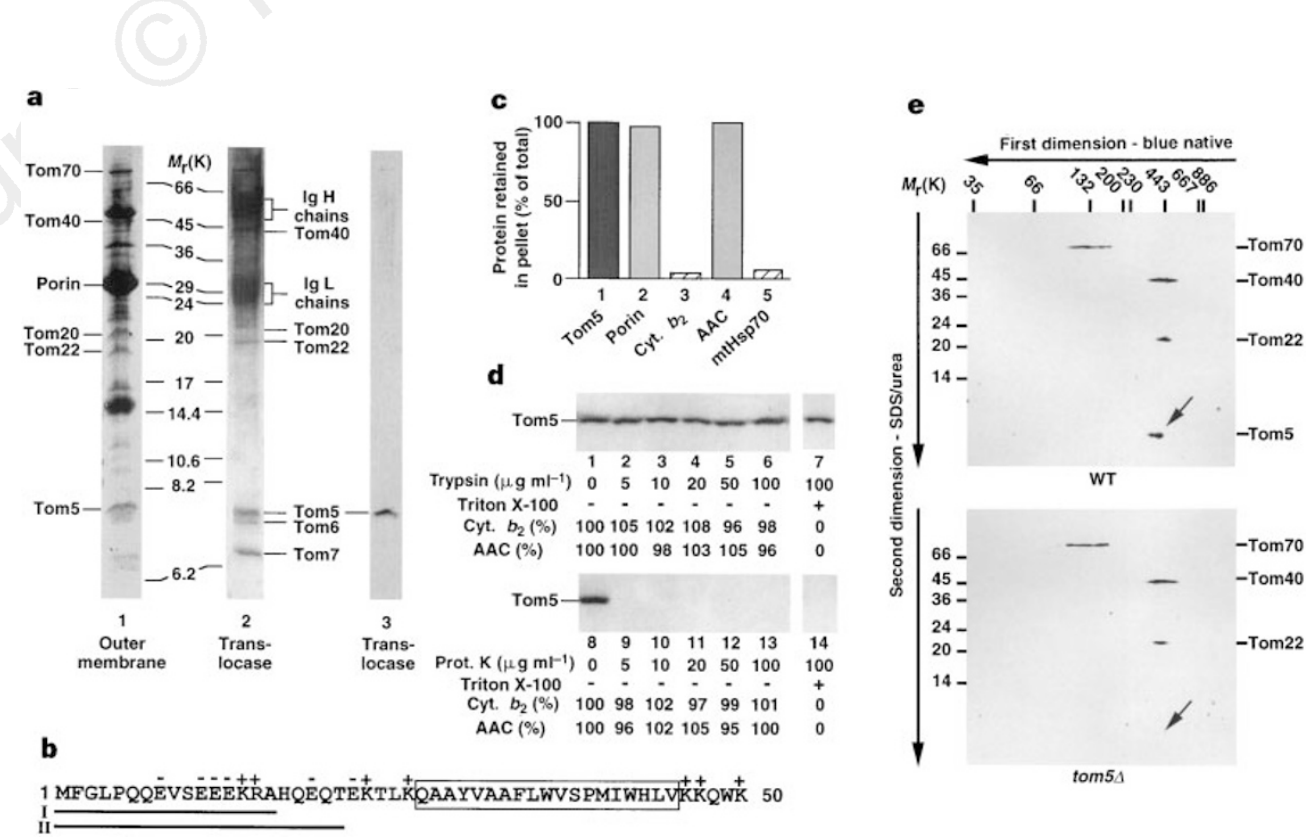

Figure 1 Tom5 is an integral mitochondrial outer membrane protein exposed to the cytosol. a, Purified outer-membrane vesicles (lane 1) and outer-membrane translocase (lane 2) stained with Coomassie brilliant blue R250. Immunoreaction of outer-membrane translocase with anti-Tom5N antibodies (lane 3). b. Primary structure of Tom5. N-terminal sequence of Tom5 derived from outer-membrane vesicles (I) or translocase (II) are underlined. The predicted membrane anchor is boxed. c, Tom 5 is not extracted at pH 11.5. Cyt. $b_{2}$, cytochrome $b_{2}$; AAC, ADP/ATP carrier. $\mathbf{d}$, The $\mathrm{N}$ terminus of Tom5 is located at the cytosolic side. Mitochondria were treated with trypsin and proteinase $\mathrm{K}$ (Prot. K) in the presence or absence of Triton X-100. e, Tom5 is present in the 400K Tom complex. Digitonin-lysed mitochondria were separated by blue native electrophoresis. WT, wild-type. Arrow, position of Tom5. 
dimer of Tom40) as described ${ }^{5,12}$. The major crosslinking product of $\sim 35 \mathrm{~K}$ was selectively recognized by antibodies directed against Tom5 (Fig. 3a, lane 2) and was absent in tom $5 \Delta$ mitochondria (Fig. 3a, lanes 7-9) (Su9-DHFR is a 29K protein). We conclude that Tom 5 is in close proximity to the accumulated preprotein. To determine the step at which Tom5 functions in protein import, Su9-DHFR was arrested at different import stages ${ }^{5,12}$. In the absence of a membrane potential, the precursor form of Su9-DHFR accumulated at the mitochondrial outer membrane (Fig. 3b, lanes 1 and 2). In the presence of a membrane potential, but at low levels
Figure 2 Tom5 is required for import of preproteins to all four mitochondrial subcompartments. a, Growth of yeast cells on fermentable (YPD) or non-fermentable (YPG) medium at $37^{\circ} \mathrm{C}$. b. Mitochondrial content of marker proteins is comparable in wildtype (WT) and tom5 $\Delta$. Hsp60 (matrix); $F_{1}$-ATPase subunit- $\beta$ ( $F_{1} \beta$; matrix side of inner membrane). c, tom $5 \Delta$ mitochondria are able to generate a membrane potential $(\Delta \psi)$ assessed by fluorescence spectrometry with the dye $\mathrm{DiSC}_{3}(5)$. dd, Deletion of TOM5 leads to severe import defects. ${ }^{35} \mathrm{~S}$-labelled preproteins were incubated with mitochondria at $25^{\circ} \mathrm{C}$. The proteinase K-treated mitochondria were analysed by SDS-PAGE and digital autoradiography. The amount of protein imported into wild-type mitochondria after $5 \mathrm{~min}$ was set to 100\% (bars, s.e.m. from 3-6 experiments). Precursor (p), intermediate (i) and mature $(\mathrm{m})$ forms of the preproteins are shown; $\alpha$-MPP, $\alpha$-subunit of mitochondrial processing peptidase.

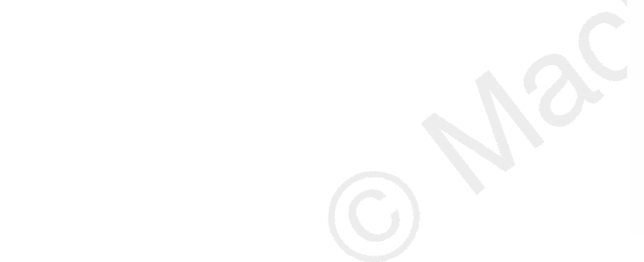

a
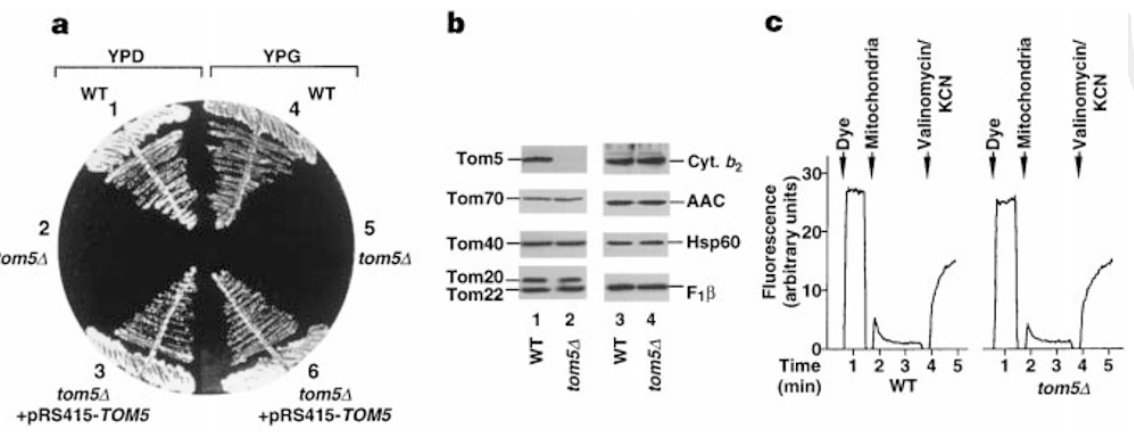

d
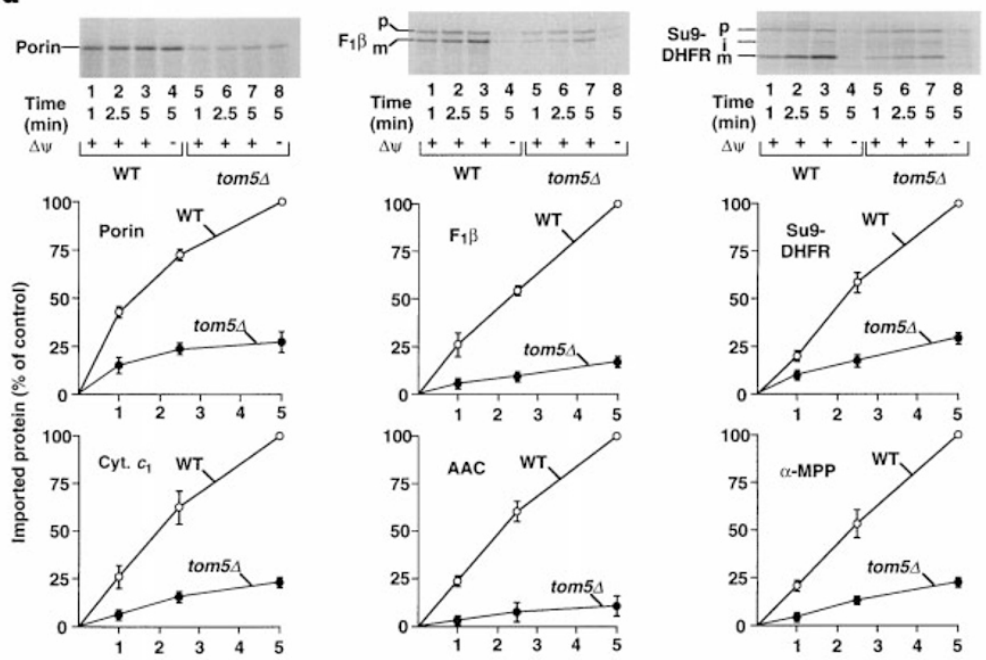

(min) 12.55 +

(min) \begin{tabular}{llllll}
1 & 2.5 & 5 & 5 & 1 & 2.55 \\
\hline
\end{tabular}

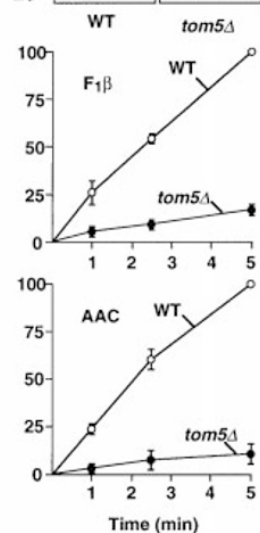

$\Delta \psi \frac{+++-L^{+}++\cdot}{W T}$
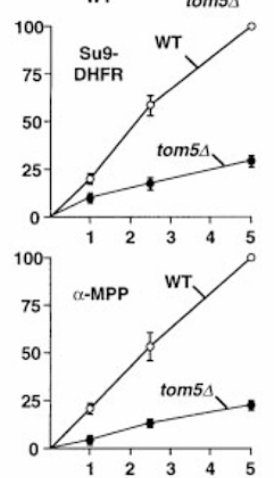

b

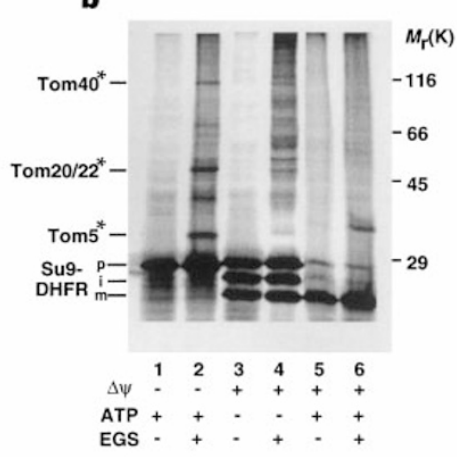

Figure 3 Crosslinking of preproteins to Tom5 depends on the import stage. a, ${ }^{35} \mathrm{~S}$-labelled precursor of Su9SHFR was incubated with isolated de-energized mitochondria. Mitochondria were reisolated, subjected to crosslinking with EGS, and analysed directly (lanes 3-10) or subjected to immunoprecipitation (lanes 1 and 2). $\mathbf{b}$, ${ }^{35}$ S-labelled Su9-DHFR was incubated with wild-type (WT) mitochondria at different import stages ${ }^{12}$ (outer membrane, lanes 1 and 2; membrane spanning, lanes 3 and 4; full import, lanes 5 and 6). c. ${ }^{35}$ S-labelled precursor of the ADP/ATP carrier was accumulated at mitochondria at different import stages ${ }^{12}$ (receptors, lanes 1 and 2; postreceptor, lanes 3-10, 13 and 14 (no protease treatment); full import, lanes 11 and 12). The efficiency of crosslinking to Tom5 (crosslinking product per accumulated preprotein) at the postreceptor stage was $\sim 2.5$-fold higher than that at the receptor stage. Asterisks indicate crosslinking products. Pre-imm., preimmune.

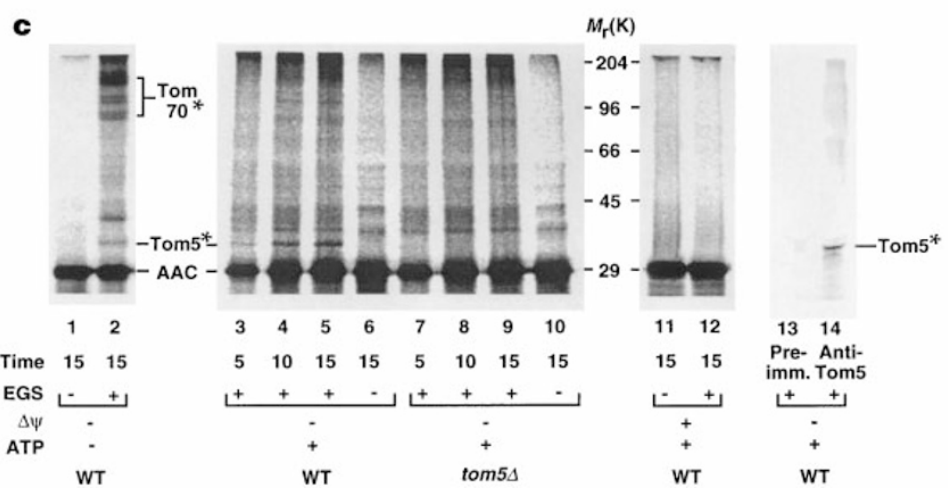


of ATP, Su9-DHFR accumulated, spanning both outer and inner membranes, leading to partial cleavage of the presequence by the matrix-processing peptidase (Fig. 3b, lanes 3 and 4). In the presence of both a membrane potential and ATP, Su9-DHFR is imported into the mitochondrial matrix and processed to the mature form (Fig. 3 b, lanes 5 and 6). Crosslinking between Su9-DHFR and Tom 5 was efficient when the preprotein was accumulated at the outer membrane (Fig. 3b, lane 2), whereas only a weak crosslinking efficiency was observed with the two-membrane-spanning intermediate (Fig. $3 \mathrm{~b}$, lane 4). As expected, no crosslinking to Tom5 occurred when Su9-DHFR was imported into the matrix (Fig. 3b, lane 6).

We investigated whether Tom 5 was also in close proximity to a non-cleavable preprotein with internal targeting information. In the absence of a membrane potential, the ADP/ATP carrier can be accumulated at two distinct stages at the outer membrane: at low levels of ATP, preferentially at receptor sites; and in the presence of ATP, preferentially at a post-receptor stage $\mathrm{e}^{10,12,17}$. In the presence of ATP, a crosslinking product between the preprotein and Tom 5 was generated in wild-type mitochondria (Fig. 3c, lanes 3-5) that was recognized by antibodies directed against Tom5 (Fig. 3c, lane 14) and was absent in tom $5 \Delta$ mitochondria (Fig. 3c, lanes 7-9). At low levels of ATP, prominent crosslinking occurred to Tom70 (mono- meric and dimeric forms ${ }^{18}$ ), whereas crosslinking to Tom 5 as weaker (Fig. 3c, lane 2). As expected, the ADP/ATP carrier imported to its functional location in the inner membrane was not crosslinked to Tom5 (Fig. 3c, lane 12). We conclude from this that Tom5 is in close proximity to both cleavable and non-cleavable preproteins that accumulated at the outer membrane. Crosslinking is most efficient at a postreceptor stage.

To determine which import stage requires Tom5, we prepared IgG directed against Tom5, and pre-bound them to isolated wild-type mitochondria. Subsequently, accumulation of the preprotein of the ADP/ATP carrier at distinct import stages was studied. Anti-Tom5 did not influence the binding of ADP/ATP carrier to receptor sites (Fig. 4a, column 3), but strongly inhibited the accumulation of ADP/ATP carrier at a postreceptor (GIP) stage of the outer membrane (Fig. 4a, column 6). Moreover, the preprotein Su9DHFR was accumulated in the precursor form at the surface of tom $5 \Delta$ mitochondria. When the mitochondria were pretreated with a low concentration of trypsin that removes the cytosolic receptor domains $^{12}$, this accumulation of pSu9-DHFR was strongly inhibited (Fig. 4b, lanes 2 and 4). We conclude that Tom5 is required for the import of cleavable and non-cleavable preproteins at a postreceptor stage. a

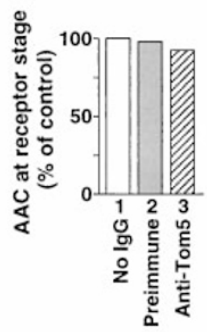

c
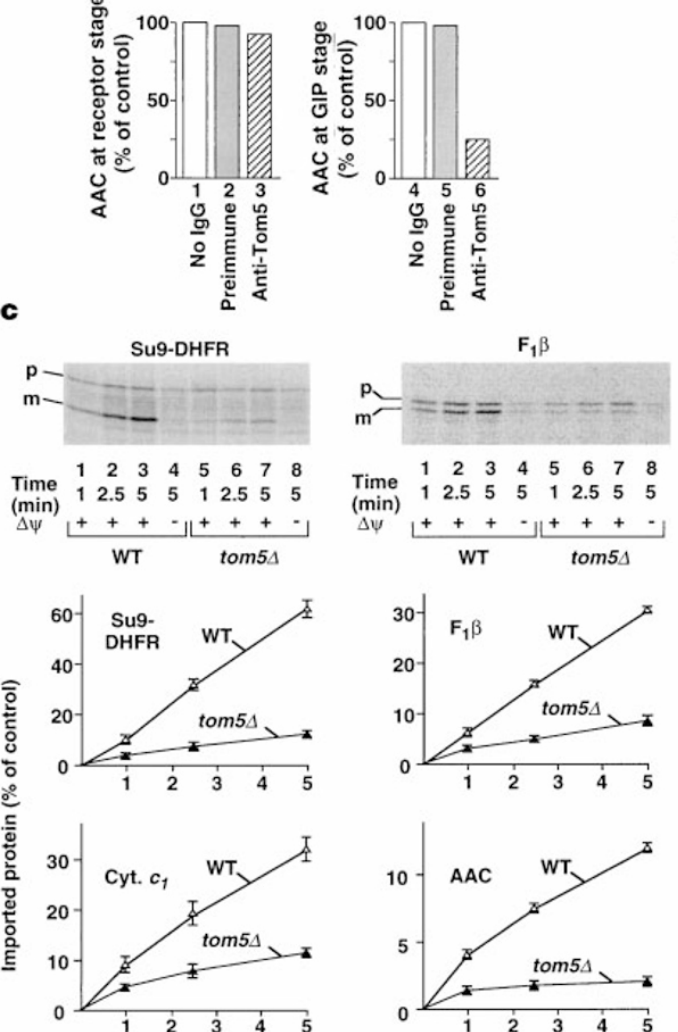

d

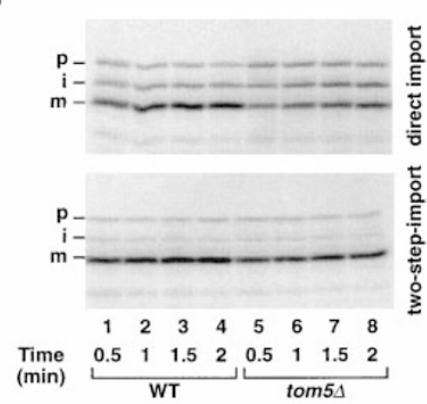

$F_{1} \beta$

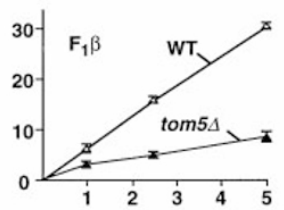

b
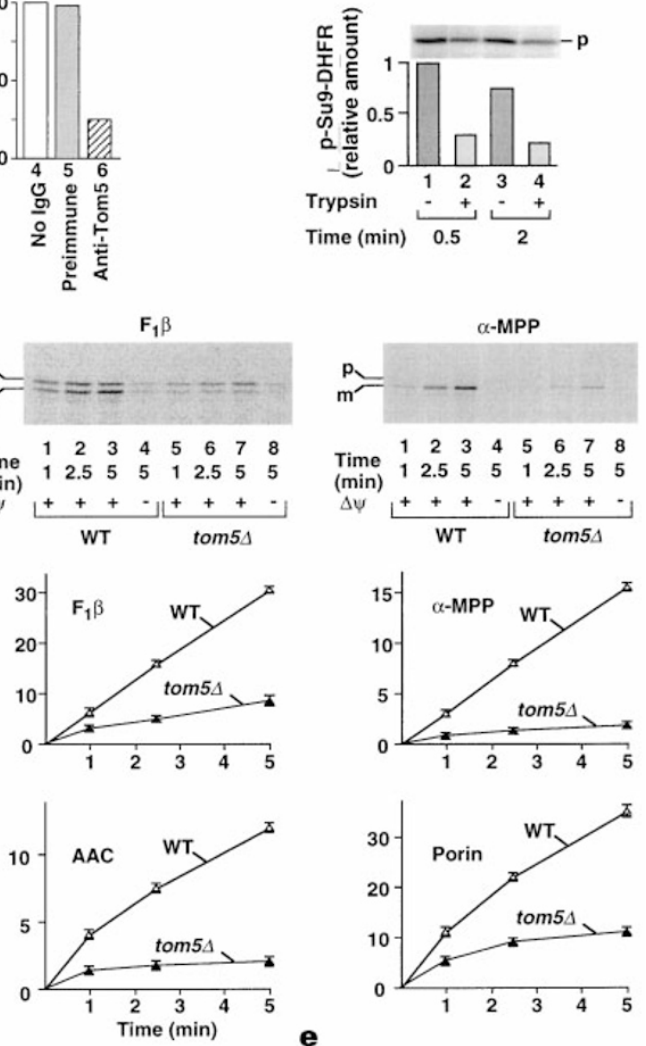

e

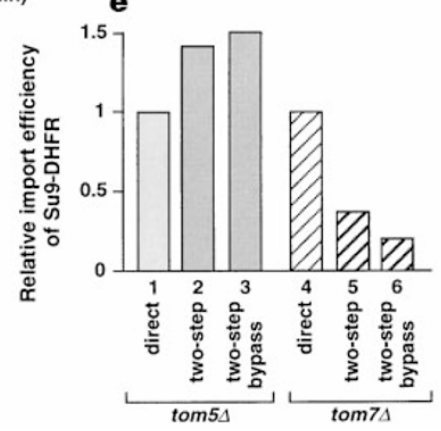

Figure 4 Tom5 functions between receptors and GIP. a, IgG (pre-immune or anti-Tom5N) were prebound to wild-type (WT) mitochondria. ADP/ATP carrier was accumulated at receptor sites (samples 1-3) or GIP (samples 4-6; protease treatment after the accumulation ${ }^{12}$ ). b. The tom5 5 mitochondria were pretreated with trypsin or mock-treated. After reisolation, mitochondria were incubated with pSu9-DHFR. c, Bypass import of preproteins is strongly impaired by lack of Tom5. Mitochondria were pretreated with trypsin. Preproteins were imported as described for Fig. 2b. The amount of import into non-trypsinized WT mitochondria after 5 min was set to $100 \%$. d, Twostep import. Su9-DHFR was bound to de-energized mitochondria. Mitochondria were reisolated and a membrane potential $(\Delta \psi)$ was generated for the times indicated. For direct import, Su9-DHFR was added in the second incubation (presence of membrane potential). e, Direct and two-step import were performed into $W T$, tom $5 \Delta$ and tom $7 \Delta$ mitochondria for $1 \mathrm{~min}$, and the import ratios between mutant and WT were determined ${ }^{11}$ (bypass, mitochondria pretreated with trypsin). The relative import efficiency of direct import into the mutant mitochondria was set to 1. Precursor (p), intermediate (i) and mature $(m)$ forms of the preproteins are shown; $\alpha$-MPP, $\alpha$ subunit of mitochondrial processing peptidase. 
The mild pretreatment of mitochondria with trypsin (5$7.5 \mu \mathrm{g} \mathrm{ml}^{-1}$ ) removes the cytosolic domains of the surface receptors Tom20-Tom 22 and Tom70-Tom37, but leaves Tom40 intact. Preproteins can still be imported into mitochondria, although with a reduced efficiency ${ }^{19}$; this 'bypass import' reflects the direct insertion of preproteins into the GIP ${ }^{9,11}$. We tested the bypass import of preproteins destined for the distinct subcompartments with wildtype and tom $5 \Delta$ mitochondria, and observed that the receptorindependent import strongly depended on the presence of Tom5 (Fig. 4c). This demonstrates that Tom 5 is required for preprotein insertion into the import pore.

When the preprotein was denatured with urea before import, the strong dependence on Tom5 was not relieved (data not shown), indicating that Tom 5 is required for import independently of the folding state of a preprotein. We then performed a two-step import of urea-denatured Su9-DHFR. This allows us to define a late function of the outer-membrane translocase in preprotein translocation. The unfolded preprotein is accumulated at the outer membrane in the absence of a membrane potential, and is subsequently imported by regeneration of a membrane potential ${ }^{11}$. Tom7 and the intermembrane space domain of Tom 22 have been found to be required for this productive accumulation of preproteins at the trans site of the outer membrane $e^{4,11,20}$ (M. Moczko, U.B., M.K., A.H. and N.P., unpublished data). This is demonstrated by a reduction of two-step import in comparison to direct import into tom $7 \Delta$ mitochondria (Fig. 4e, compare columns 5 and 4). The efficiency of two-step import into tom $5 \Delta$ mitochondria was not decreased in comparison to direct import (Fig. 4d, lanes 5-8; Fig. 4e, columns 2 and 1). To exclude an involvement of the surface receptor domains in the productive accumulation, the two-step import reaction was performed with trypsinized mitochondria ${ }^{11}$ (Fig. 4e, columns 3 and 6 ). In contrast to the strong inhibition in tom $7 \Delta$ mitochondria (Fig. 4e, column 6), a lack of Tom5 did not inhibit the productive accumulation of the preprotein at the outer-membrane trans site (Fig. 4e, column 3). Thus Tom 5 is not required at this late stage of preprotein transport across the outer membrane; it functions between surface receptors and the import pore.

With tom $5 \Delta$ mitochondria the $\mathrm{N}$-terminal segment of Tom 40 , which is exposed to the cytosolic side, was cleaved off by trypsin $\left(>15 \mu \mathrm{g} \mathrm{ml}^{-1}\right.$ ), whereas in wild-type mitochondria Tom40 was protected against tryptic attack (data not shown). This finding suggests that Tom5 is closely associated with the $\mathrm{N}$ terminus of Tom 40 and, because of its own trypsin resistance (Fig. 1d, it protects Tom 40 from tryptic digest.

To assess the in vivo relevance of Tom 5 in cooperation with the other Tom proteins, two genetic approaches were used. First, overexpression of each of the essential Tom proteins, Tom 40 and Tom22, did not suppress the defect caused by deletion of TOM5, indicating that Tom 5 has a function different from that of Tom 22 and Tom 40. Second, synthetic lethality is observed when mutations or deletions of two genes together cause lethality, whereas cells carrying the mutation or deletion of either gene alone are viable ${ }^{11,12,21}$. Spores containing double deletions, tom $5 \Delta$ combined with every viable deletion of the other TOM genes (tom6 $\Delta$, tom $7 \Delta$, tom $20 \Delta$, tom $37 \Delta$ and tom $70 \Delta$ ), were inviable, demonstrating synthetic lethality. Only the double deletion tom $5 \Delta$ tom $72 \Delta$ was viable (Tom72 is a Tom70homologue of unknown function that loosely assembles with the Tom machinery ${ }^{2}$ ). These genetic results are indicative of a functional interaction between Tom 5 and most other Tom proteins.

Tom5 is required for the import of preproteins into mitochondria. The Tom proteins identified so far function as receptors, import pore components or modulators of translocase dynamics, but Tom 5 provides a new functional element. It represents a central link between the initial recognition event of preproteins (Tom70Tom 37 and/or Tom20-Tom22) and the passage across the membrane (Tom40). By studying a preprotein targeted through Tom70, Tom 22 had been proposed to represent the link between import receptors and $\mathrm{GIP}^{22}$, yet it was found that Tom 22 is an essential subunit of the import receptor Tom22-Tom20 (refs 4-6). Preproteins initially recognized by Tom70-Tom 37 are transferred to the second receptor subcomplex Tom22-Tom 20 before transfer into $\mathrm{GIP}^{23,24}$, explaining the observation of an action of Tom 22 between the receptor Tom70 and GIP. Our results show that, after convergence of the targeting pathways at Tom 20-Tom22, the preproteins are transferred to Tom5. The negative net charge of its cytosolic segment should facilitate interaction with positively charged targeting sequences. We propose that the cytosolic segment of Tom 5 helps to insert preproteins into the GIP. Tom5 thus functions as a mediator between receptors and GIP, and may also represent an early component of the translocation machinery (that is, the GIP) itself. Small subunits have also been identified in other preprotein translocases, such as Sss1/Sec61- $\gamma$ of the endoplasmic rediculum and SecE in the bacterial export system ${ }^{25-27}$. Tom 5 is the smallest of the known cellular preprotein translocase components, and is the only small component $(<10 \mathrm{~K})$ for which evidence of a direct interaction with preproteins exists (the $11 \mathrm{~K}$ protein Tim 11 of the mitochondrial inner membrane has also been crosslinked to a preprotein in transit ${ }^{28}$ ). The work on Tom6 and Tom7 supported the view of an indirect role of small subunits in preprotein translocases, but the findings made for Tom5 should stimulate investigations on a direct involvement of small subunits in preprotein transport with other translocases as well.

\section{Methods}

Cloning and disruption of TOM5. A 1,200-base pair genomic fragment of TOM5 was amplified by PCR, digested with EcoRI and Pst $\mathrm{I}$, and cloned into pGEM4Z. Inverse PCR mutagenesis was used to delete the TOM5 ORF and to introduce a SmaI and SalI site. After digestion with SmaI and SalI, the marker HIS 3 was introduced. The plasmid was linearized with EcoRI and used to transform the diploid yeast strain YPH501 (ref. 11). His $3^{+}$colonies were selected and allowed to sporulate.

Isolation and characterization of mitochondria and translocase. Isolation of $S$. cerevisiae mitochondria, the outer-membrane translocase (coprecipitation with antibodies directed against Tom 40 from digitonin-lysed mitochondria), and outer-membrane vesicles were performed as described ${ }^{11,12}$. Antisera against an oligopeptide corresponding to the $12 \mathrm{~N}$-terminal residues of Tom5 were prepared by coupling the peptide to keyhole limpet haemocyanin and injection into rabbits. The amino-acid sequence analysis of Tom 5 was performed as described $^{5,11,29}$. For analysis of the Tom complex by two-dimensional gel analysis, $100 \mu \mathrm{g}$ of wild-type or tom $5 \Delta$ mitochondria were lysed in $1 \%$ digitonin-containing buffer and, for the first dimension, separated by blue native electrophoresis ${ }^{14,30}$. Individual lanes were excised and analysed by ureaSDS-PAGE as second dimension, and immunoblotted onto nitrocellulose. Mitochondria $(100 \mu \mathrm{g}$ mitochondrial protein) were treated at $\mathrm{pH} 11.5$ with $100 \mathrm{mM} \mathrm{Na} \mathrm{CO}_{3}$ and separated into supernatant and pellet, followed by immunodecoration ${ }^{11}$.

Protein import and crosslinking. Preproteins were synthesized in rabbit reticulocyte lysates in the presence of $\left[{ }^{35} \mathrm{~S}\right]$ methionine/ $\left[{ }^{35} \mathrm{~S}\right]$ cysteine ${ }^{12}$. Import reactions into isolated mitochondria were performed at $25^{\circ} \mathrm{C}$ as described ${ }^{11}$. To allow direct comparison of import reactions, the amounts of preproteins and mitochondria were the same in parallel reactions, and quantifications were made from the linear import ranges ${ }^{12}$. The amounts of imported proteins were determined by treatment of mitochondria with proteinase $\mathrm{K}$ after the import reaction, followed by separation on SDS-PAGE and digital autoradiography. Surface domains of receptors were removed by a treatment of mitochondria before the import reaction with $5-7.5 \mu \mathrm{g} \mathrm{ml}^{-1} \operatorname{trypsin}^{11}$. IgG (from preimmune serum or anti-Tom5 $\mathrm{N}$ serum) was isolated and incubated with isolated mitochondria ( $150 \mu \mathrm{g}$ IgG per sample) as described ${ }^{12}$. For accumulation of translocation intermediates, ATP was depleted (by apyrase) and/or the membrane potential was dissipated ${ }^{12}$. For two-step import ${ }^{11}$, the membrane potential of mitochondria was dissipated by valinomycin in the presence of potassium, and the urea-denatured preprotein Su9-DHFR was added. Mitochondria were reisolated and a membrane potential was re-established by resuspension in buffer with valinomycin, but without potassium. For direct 
import, the precursor was added at the second incubation step. For crosslinking, mitochondria containing accumulated ${ }^{35} \mathrm{~S}$-labelled preproteins were re-isolated through a sucrose cushion ${ }^{5,12}$, washed and incubated with EGS. After quenching of the crosslinker with Tris buffer, the proteins were precipitated with trichloroacetic acid. The samples were either analysed by SDSPAGE or lysed in SDS-containing buffer and immunoprecipitated in Triton $\mathrm{X}$-100-containing buffer ${ }^{12}$. Standard procedures were used for immunoblotting onto nitrocellulose. Bands were detected by the Enhanced Chemiluminescence System (Amersham). Membrane potential was assessed using $\operatorname{DiSC}_{3}(5)$ (Molecular Probes) $)^{15,16}$.

Received 24 February; accepted 7 May 1997

1. Schatz, G. \& Dobberstein, B. Common principles of protein translocation across membranes. Science 271, 1519-1526 (1996)

2. Pfanner, N. \& Meijer, M. Mitochondrial biogenesis: the Tom and Tim machine. Curr. Biol. 7, R100R103 (1997).

3. Lill, R. \& Neupert, W. Mechanisms of protein import across the mitochondrial outer membrane. Trends Cell Biol. 6, 56-61 (1996).

4. Bolliger, L., Junne, T., Schatz, G. \& Lithgow, T. Acidic receptor domains on both sides of the outer membrane mediate translocation of precursor proteins into yeast mitochondria. $E M B O J .14,6318$ 6326 (1995).

Hönlinger, A. et al. The mitochondrial receptor complex: Mom22 is essential for cell viability and directly interacts with preproteins. Mol. Cell. Biol. 15, 3382-3389 (1995).

6. Mayer, A., Nargang, F. E., Neupert, W. \& Lill, R. MOM22 is a receptor for mitochondrial targetin sequences and cooperates with MOM19. EMBO J. 14, 4204-4211 (1995).

7. Gratzer, S. et al. Mas37p, a novel receptor subunit for protein import into mitochondria. J. Cell Biol. 129, 25-34 (1995)

8. Vestweber, D., Brunner, J., Baker, A. \& Schatz, G. A $42 \mathrm{~K}$ outer-membrane protein is a component of the yeast mitochondrial protein import site. Nature 341, 205-209 (1989).

9. Kiebler, M. et al. Identification of a mitochondrial receptor complex required for recognition and membrane insertion of precursor proteins. Nature 348, 610-616 (1990).

10. Alconada, A., Kübrich, M., Moczko, M., Hönlinger, A. \& Pfanner, N. The mitochondrial receptor complex: the small subunit Mom8b/Isp6 supports association of receptors with the general insertion pore and transfer of preproteins. Mol. Cell. Biol. 15, 6196-6205 (1995).

11. Hönlinger, A. et al. Tom7 modulates the dynamics of the mitochondrial outer membrane translocase and plays a pathway-related role in protein import. EMBO J. 15, 2125-2137 (1996)

12. Alconada, A., Gärtner, F., Hönlinger, A., Kübrich, M. \& Pfanner, N. Mitochondrial receptor complex from Neurospora crassa and Saccharomyces cerevisiae. Meth. Enzymol. 260, 263-286 (1995).

13. Steger, H. F. et al. Import of ADP/ATP carrier into mitochondria: two receptors act in parallel. J. Cell Biol. 111, 2353-2363 (1990).

14. Dekker, P. J. T., Müller, H., Rassow, J. \& Pfanner, N. Characterization of the preprotein translocase of the outer mitochondrial membrane by blue native electrophoresis. Biol. Chem. 377, 535-538 (1996).

15. Eilers, M., Oppliger, W. \& Schatz, G. Both ATP and an energized inner membrane are required to import a purified precursor protein into mitochondria. EMBO J. 6, 1073-1077 (1987).

16. Gärtner, F. et al. Mitochondrial import of subunit Va of cytochrome $c$ oxidase characterized with yeas mutants: indpendence from receptors, but requirement for matrix hsp70 translocase function. J. Biol. Chem. 270, 3788-3795 (1995).

17. Pfanner, N., Tropschug, M. \& Neupert, W. Mitochondrial protein import: nucleoside triphosphates are involved in conferring import-competence to precursors. Cell 49, 815-823 (1987).

18. Söllner, T. et al. Mapping of the protein import machinery in the mitochondrial outer membrane by rosslinking of translocation intermediates. Nature 355, 84-87 (1992).

19. Pfaller, R., Pfanner, N. \& Neupert, W. Mitochondrial protein import: bypass of proteinaceous surface receptors can occur with low specificity and efficiency. J. Biol Chem. 264, 34-39 (1989).

20. Mayer, A., Neupert, W. \& Lill, R. Mitochondrial protein import: reversible binding of the presequence at the trans side of the outer membrane drives partial translocation and unfolding. Cell 80, 127-137 (1995)

21. Huffaker, T. C., Hoyt, M. A. \& Botstein, D. Genetic analysis of the yeast cytoskeleton. Annu. Rev. Genet 21, 259-284 (1987)

22. Kiebler, M. et al. The mitochondrial receptor complex: a central role of MOM22 in mediating preprotein transfer from receptors to the general insertion pore. Cell 74, 483-492 (1993).

23. Haucke, V., Horst, M., Schatz, G. \& Lithgow, T. The Mas20p and Mas70p subunits of the protein import receptor of yeast mitochondria interact via the tetratricopeptide repeat motif in Mas20p: evidence for a single hetero-oligomeric receptor. EMBO J. 15, 1231-1237 (1996).

24. Mihara, K. \& Omura, T. Cytoplasmic chaperones in precursor targeting to mitochondria: the role of MSF and hsp70. Trends Cell Biol. 6, 104-108 (1996).

25. Brundage, L., Fimmel, C. J., Mizushima, S. \& Wickner, W. SecY, SecE, and Band 1 form the membrane-embedded domain of Escherichia coli preprotein translocase. J. Biol. Chem. 267, 41664170 (1992).

26. Esnault, Y., Feldheim, G., Blondel, M. O., Schekman, R. \& Képès, F. SSS1 encodes a stabilizing component of the Sec61 subcomplex of the yeast protein translocation apparatus. J. Biol. Chem. 269, 27478-27485 (1994).

27. Rapoport, T. A., Jungnickel, B. \& Kutay, U. Protein transport across the eukaryotic endoplasmic reticulum and bacterial inner membranes. Annu. Rev. Biochem. 65, 271-303 (1996).

28. Tokatlidis, K. et al. Translocation arrest of an intramitochondrial sorting signal next to Tim11 at the inner-membrane import site. Nature 384, 585-588 (1996).

29. Eckerskorn, C., Mewes, W., Goretzki, H. \& Lottspeich, F. A new siliconized-glass fiber as support for protein-chemical analysis of electroblotted proteins. Eur. J. Biochem. 176, 509-519 (1988)

30. Schägger, H., Cramer, W. A. \& von Jagow, G. Analysis of molecular masses and oligomeric states of protein complexes by blue native electrophoresis and isolation of membrane protein complexes by two-dimensional native electrophoresis. Analyt. Biochem. 217, 220-230 (1994).

Acknowledgements. We thank M. Ryan for comments on the manuscript. This work was supported by the Deutsche Forschungsgemeinschaft, Sonderforschungsbereich 388 Freiburg, the Fonds der Chemischen Industrie (N.P.) and a long-term fellowship from the Human Frontier Science Program mischen 1 (P.J.T.D.).
(

Correspondence and requests for materials should be addressed to N.P. (e-mail: pfanner@ruf.unifrieburg.de). The sequence of Tom5 has been deposited in the Swiss-Prot protein sequence database, accession no. P80967.

\section{X-chromosome-counting mechanisms that determine nematode sex}

\author{
Monique Nicoll, Chantal C. Akerib \& Barbara J. Meyer
}

Department of Molecular and Cell Biology, University of California at Berkeley, Berkeley, California 94720-3204, USA

Sex is determined in Caenorhabditis elegans by an X-chromosome-counting mechanism that reliably distinguishes the twofold difference in X-chromosome dose between males (1X) and hermaphrodites $(2 \mathrm{X})^{1,2}$. This small quantitative difference is translated into the 'on/off' response of the target gene, xol-1, a switch that specifies the male fate when active and the hermaphrodite fate when inactive ${ }^{3}$. Specific regions of $X$ contain counted signal elements whose combined dose sets the activity of xol-1 (ref. 4). Here we ascribe the dose effects of one region to a discrete, protein-encoding gene, fox-1. We demonstrate that the dosesensitive signal elements on chromosome $\mathrm{X}$ control xol-1 through two different molecular mechanisms. One involves the transcriptional repression of $x o l-1$ in $\mathrm{XX}$ animals. The other uses the putative RNA-binding protein encoded by fox-1 to reduce the level of $x o l-1$ protein. These two mechanisms of repression act together to ensure the fidelity of the $\mathrm{X}$-chromosome counting process.

The control of sex by the number of $\mathrm{X}$ chromosomes was the first genetic regulatory mechanism to be described ${ }^{5}$. In the eighty-one years since its discovery, the mechanism by which chromosomes are counted to determine sex has been established only for Drosophila ${ }^{6}$. In C. elegans, the xol-1 gene interprets X-chromosome number ${ }^{3,4}$. Thus, the question of how the worm counts its X chromosomes has been reduced to the problem of identifying the elements that control xol-1 activity and the mechanism by which they do so. Sex-specific regulation of xol-1 is reflected in the tenfold-higher level of xol-1 transcripts in males than in hermaphrodites ${ }^{3}$. Setting xol-1 expression is essential for viability as well as sex determination because $x o l$ 1 also controls X-chromosome dosage compensation ${ }^{7}$. Dosage compensation equalizes X-chromosome expression between the sexes by halving the level of transcripts made from each $\mathrm{X}$ chromosome in hermaphrodites ${ }^{6,8}$. Inappropriate activation of $x o l-1$ in XX animals prevents the reduction of $\mathrm{X}$ expression and thereby causes hermaphrodite-specific lethality ${ }^{3}$. Repression of $x o l-1$ expression in $\mathrm{XO}$ animals causes inappropriate reduction of $\mathrm{X}$ expression and hence male-specific lethality ${ }^{3,7}$

To understand how the X-chromosome signal elements regulate xol-1, we first investigated whether the sex-specific difference in $x o l$ 1 transcript levels results from transcriptional regulation. The activity of a lac $Z$ reporter gene under the transcriptional control of the xol-1 promoter (Fig. 1g) was assayed in males and hermaphrodites. We observed that XO animals carrying an integrated array ( $y$ Is33) containing multiple copies of the Pxol-1::lacZ reporter transgene had high $\beta$-galactosidase activity (Fig. 1c) and that array-bearing XX animals had low activity (Fig. 1b), indicating that transcriptional regulation is central to the sex-specific expression of xol-1.

Our previous genetic analysis demonstrated that X-chromosome dose is communicated to $x o l-1$ by elements in at least three distinct chromosomal regions ${ }^{4}$ (Fig. 1a). Duplication of all three regions causes $>99 \%$ XO-specific lethality ${ }^{4}$. This lethality is suppressed by constitutive expression of xol-l. Deletion of one copy of all three regions in XX animals causes lethality and severe dosage compensation defects that are suppressed by $x o l-1$ null mutations ${ }^{4}$. To understand how individual components of the signal regulate $x o l-1$, we asked whether deletions that eliminate $\mathrm{X}$ signal elements affect 